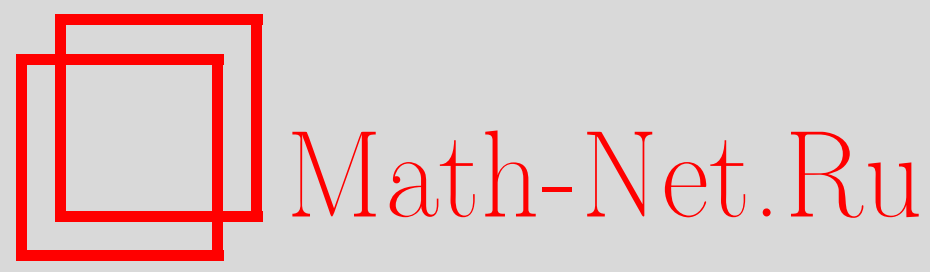

M. Е. Чанга, О нижних оценках модуля дзета-функции Римана на критической прямой, Матем. заметки, 2004, том 76, выпуск 6, 922-927

DOI: https://doi.org/10.4213/mzm151

Использование Общероссийского математического портала Math-Net.Ru подразумевает, что вы прочитали и согласны с пользовательским соглашением http://www.mathnet.ru/rus/agreement

Параметры загрузки:

IP: 52.90 .164 .192

26 апреля 2023 г., $14: 28: 58$

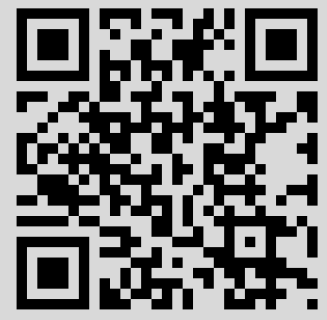


УДК 511

\section{О НИЖНИХ ОЦЕНКАХ МОДУЛЯ ДЗЕТА-ФУНКЦИИ РИМАНА НА КРИТИЧЕСКОЙ ПРЯМОЙ}

\section{М.Е. Чанга}

Установлена связь между нижней оценкой максимума модуля $\zeta(1 / 2+i T+s)$ в круге $|s| \leqslant H$ и нижней оценкой максимума модуля $\zeta(1 / 2+i T+i t)$ на отрезке $|t| \leqslant H$ при $0<H(T) \leqslant 1 / 2$. Доказана теорема о нижней оценке максимума модуля $\zeta(1 / 2+i T+i t)$ на отрезке $|t| \leqslant H$ при $40 \leqslant H(T) \leqslant \log \log T$.

Библиограффия: 6 названий.

1. Введение. Формулировка результатов. В статье продолжены исследования функций $F(T ; H)$ и $G(T ; H)$, которые ввел Карацуба в [1] и [2]:

$$
F(T ; H)=\max _{|t-T| \leqslant H}\left|\zeta\left(\frac{1}{2}+i t\right)\right|, \quad G(T ; H)=\max _{|s| \leqslant H}\left|\zeta\left(\frac{1}{2}+i T+s\right)\right| .
$$

Здесь устанавливается связь между нижними оценками $F(T ; H)$ и $G(T ; H)$ при $0<$ $H \leqslant 1 / 2$ и доказьвается теорема о нижней оценке $F(T ; H)$ при $H \geqslant 40$. В статье употребляются общепринятые обозначения; в частности, $c_{1}, c_{2}, \ldots$ - положительные абсолютные постоянные.

Tеорема 1. Пусть $0<H(T) \leqslant 1 / 2$ и при $T \geqslant T_{1}>0$ имеет место оченка

$$
G(T ; H)>T^{-c_{1}} \text {. }
$$

Тогда при $T \geqslant T_{2}>0$ справедлива оченка

$$
F(T ; H)>T^{-c_{2}} .
$$

ЗАмечание 1. Утверждение теоремы при $H=(\log T)^{-1}$ доказано Гараевым в [3].

ЗАМЕчАнИЕ 2. Из теоремы следует, что гипотезы 1-3 из [1] эквивалентны гипотезам $1-3$ из [2].

Tеорема 2. Пусть $40 \leqslant H(T) \leqslant \log \log T$. Тогда при $T \geqslant T_{1}>0$ справедлива оценка

$$
F(T ; H)>T^{-\exp (-0.1 H)} .
$$


ЗАМЕчАниЕ 3 . При больших $H$, а именно, $\log \log T \ll H \ll T$, нижняя оценка вида

$$
F(T ; H) \geqslant \exp \left(\frac{3}{4} \sqrt{\frac{\log H}{\log \log H}}\right)
$$

получена Баласубраманияном в [4].

ЗАмечАние 4 . При $H \gg 1$ из теоремы Титчмарша [5, с. 217] следует, что

$$
F(T ; H) \geqslant T^{-c_{1}} .
$$

ЗАмЕчАниЕ 5. Лучшей, чем (1), оценкой является неопубликованная оценка Карацубы

$$
F(T ; H) \geqslant T^{-H^{-2}} .
$$

Доказательства обеих теорем, приведенные в следующих пунктах, основаны на принципе максимума модуля и используют простейшие теоретико-функциональные свойства $\zeta(s)$ такие, как степенной порядок роста в критической полосе и ограниченность на прямой $\operatorname{Re} s=a>1$. Таким образом, эти же соображения могут быть применены и к другим аналитическим функциям, например, к $L$-функциям Дирихле и дзета-функциям Эпштейна.

Нам понадобится следующая теоретико-функциональная лемма, доказательство которой приведено, например, в [6, с. 456].

Лемма (теорема Адамара о трех кругах). Пусть $0<r_{1}<r_{2} u$ функиия $f(s)$ однозначна и аналитична в кольче $r_{1} \leqslant|s| \leqslant r_{2}$. Если

$$
M(r)=\max _{|s|=r}|f(s)|,
$$

то при $r_{1} \leqslant r \leqslant r_{2}$ имеет место оченка

$$
M(r) \leqslant\left(M\left(r_{1}\right)\right)^{\left(\log \left(r_{2} / r\right)\right) /\left(\log \left(r_{2} / r_{1}\right)\right)}\left(M\left(r_{2}\right)\right)^{\left(\log \left(r / r_{1}\right)\right) /\left(\log \left(r_{2} / r_{1}\right)\right)} .
$$

2. Доказательство теоремы 1. Положим $r_{1}=H^{3}, r_{2}=1$ и $r=H$. Тогда

$$
\frac{\log \left(r_{2} / r\right)}{\log \left(r_{2} / r_{1}\right)}=\frac{\log H^{-1}}{\log H^{-3}}=\frac{1}{3}, \quad \frac{\log \left(r / r_{1}\right)}{\log \left(r_{2} / r_{1}\right)}=\frac{\log H^{-2}}{\log H^{-3}}=\frac{2}{3} .
$$

Применяя теорему Адамара о трех кругах к функции $\zeta(1 / 2+i T+s)$, получим

$$
M(H) \leqslant M^{1 / 3}\left(H^{3}\right) M^{2 / 3}(1) .
$$

По условию при $T \geqslant T_{1}>0$

$$
M(H)=G(T ; H)>T^{-c_{1}} .
$$

С другой стороны, при $T \geqslant T_{3}>0$

$$
M(1)=G(T ; 1) \leqslant T^{c_{3}} .
$$


Таким образом, при $T \geqslant T_{2}=\max \left(T_{1}, T_{3}\right)>0$ справедлива оценка

$$
M\left(H^{3}\right)=G\left(T ; H^{3}\right)>T^{-3 c_{1}-2 c_{3}}
$$

Это означает, что на окружности $|s|=H^{3}$ существует точка $s_{0}=\sigma_{0}+i t_{0}$ такая, что

$$
\left|\zeta\left(\frac{1}{2}+i T+s_{0}\right)\right|>T^{-3 c_{1}-2 c_{3}} .
$$

Будем считать, что $\sigma_{0} \neq 0$, так как в противном случае утверждение теоремы тривиально следует из этого неравенства.

Рассмотрим функцию

$$
f(s)=\frac{1}{H^{4}}\left(s^{2}-2 H^{2} s\right)
$$

ее действительная часть имеет вид

$$
\operatorname{Re} f(s)=-\frac{t^{2}}{H^{4}}+\frac{1}{H^{4}} \sigma\left(\sigma-2 H^{2}\right) .
$$

Заметим, что функция $\sigma\left(\sigma-2 H^{2}\right)$ неположительна и монотонно убывает при $0 \leqslant \sigma \leqslant H^{2}$. Выберем прямоугольный контур $\Gamma$ с вершинами в точках $\pm i H^{2}, H^{2} \pm i H^{2}$. Очевидно выполнение неравенств

$$
\begin{array}{ll}
\operatorname{Re} f(s) \leqslant 0, & s \in \Gamma, \quad \sigma=0, \\
\operatorname{Re} f(s) \leqslant-1, & s \in \Gamma, \quad \sigma>0 .
\end{array}
$$

Точка $\left|\sigma_{0}\right|$ попадает внутрь контура $Г$. Поскольку $\left|\sigma_{0}\right| \leqslant H^{3} \leqslant \frac{1}{2} H^{2}$ имеем также неравенство

$$
\operatorname{Re} f\left(\left|\sigma_{0}\right|\right) \geqslant \operatorname{Re} f\left(\frac{1}{2} H^{2}\right)=-\frac{3}{4} .
$$

Рассмотрим теперь функцию

$$
F(s)=\zeta\left(\frac{1}{2}+i\left(T+t_{0}\right)+s \operatorname{sign} \sigma_{0}\right) T^{12\left(c_{1}+c_{3}\right) f(s)}
$$

которая аналитична в области, ограниченной контуром Г. Пользуясь неравенствами (3) и (6), находим

$$
\left|F\left(\left|\sigma_{0}\right|\right)\right|>T^{-3 c_{1}-2 c_{3}} \cdot T^{-9\left(c_{1}+c_{3}\right)}=T^{-12 c_{1}-11 c_{3}} .
$$

С другой стороны, поскольку при $s$, принадлежащем $\Gamma$,

$$
\left|i t_{0}+s \operatorname{sign} \sigma_{0}\right| \leqslant H^{3}+2 H^{2} \leqslant \frac{1}{8}+\frac{1}{2} \leqslant 1
$$

пользуясь неравенствами (2) и (5), находим

$$
\max _{s \in \Gamma, \sigma>0}|F(s)| \leqslant T^{c_{3}} \cdot T^{-12\left(c_{1}+c_{3}\right)}=T^{-12 c_{1}-11 c_{3}} .
$$


Таким образом, имеет место неравенство

$$
\max _{s \in \Gamma, \sigma>0}|F(s)|<\left|F\left(\left|\sigma_{0}\right|\right)\right|
$$

откуда получаем

$$
\left|F\left(\left|\sigma_{0}\right|\right)\right| \leqslant \max _{s \in \Gamma, \sigma=0}|F(s)| .
$$

Действительно, в противном случае имело бы место неравенство

$$
\left|F\left(\left|\sigma_{0}\right|\right)\right|>\max _{s \in \Gamma}|F(s)|
$$

которое противоречит принципу максимума модуля. Наконец, пользуясь неравенствами (4) и (7), имеем при $T \geqslant T_{2}>0$

$$
T^{-12 c_{1}-11 c_{3}}<\left|F\left(\left|\sigma_{0}\right|\right)\right| \leqslant \max _{|t| \leqslant H^{2}}|F(i t)| \leqslant \max _{|t| \leqslant H^{2}}\left|\zeta\left(\frac{1}{2}+i\left(T+t_{0}+t\right)\right)\right| .
$$

Этим доказательство завершается, ибо $\left|t_{0}+t\right| \leqslant H^{3}+H^{2} \leqslant H / 4+H / 2 \leqslant H$.

3. Доказательство теоремы 2. Положим $n=[H /(4 e \sqrt{2})] \geqslant 2$ и рассмотрим функцию

$$
f(s)=-\frac{2^{2 n}(4 n) !}{H^{4 n}}\left(\sum_{k=1}^{2 n} \frac{(-1)^{k} s^{2 k}}{2^{k}(2 k) !}+s\right)
$$

ее действительная часть имеет вид

$$
\begin{aligned}
\operatorname{Re} f(s) & =-\frac{2^{2 n}(4 n) !}{H^{4 n}}\left(\sum_{k=1}^{2 n} \frac{(-1)^{k}}{2^{k}(2 k) !} \sum_{m=0}^{k} C_{2 k}^{2 m}(-1)^{m} t^{2 m} \sigma^{2 k-2 m}+\sigma\right) \\
& =-\frac{t^{4 n}}{H^{4 n}}-\frac{2^{2 n}(4 n) !}{H^{4 n}} \sum_{m=1}^{2 n-1}(-1)^{m} t^{2 m} \sum_{k=m}^{2 n} \frac{(-1)^{k} \sigma^{2 k-2 m}}{2^{k}(2 k) !} C_{2 k}^{2 m}+f(\sigma) \\
& =-\frac{t^{4 n}}{H^{4 n}}+\sum_{m=1}^{2 n-1} \frac{t^{2 m}}{2^{m}(2 m) !}\left(-\frac{2^{2 n}(4 n) !}{H^{4 n}} \sum_{k=0}^{2 n-m} \frac{(-1)^{k} \sigma^{2 k}}{2^{k}(2 k) !}\right)+f(\sigma) .
\end{aligned}
$$

Заметим, что при $0 \leqslant \sigma \leqslant 2$ последовательность $\sigma^{2 k} /\left(2^{k}(2 k) !\right)$ монотонно убывает. Действительно, при $k \geqslant 0$ имеем

$$
\frac{\sigma^{2(k+1)}}{2^{k+1}(2 k+2) !}=\frac{\sigma^{2 k}}{2^{k}(2 k) !} \cdot \frac{\sigma^{2}}{2(2 k+1)(2 k+2)} \leqslant \frac{\sigma^{2 k}}{2^{k}(2 k) !} .
$$

Следовательно, при $0 \leqslant \sigma \leqslant 2$ справедливы оценки

$$
\frac{2^{2 n}(4 n) !}{H^{4 n}}\left(-\sigma+\frac{\sigma^{2}}{4}-\frac{\sigma^{4}}{96}\right) \leqslant f(\sigma) \leqslant \frac{2^{2 n}(4 n) !}{H^{4 n}}\left(-\sigma+\frac{\sigma^{2}}{4}\right) \leqslant 0,
$$

а также

$$
-\frac{2^{2 n}(4 n) !}{H^{4 n}} \sum_{k=0}^{2 n-m} \frac{(-1)^{k} \sigma^{2 k}}{2^{k}(2 k) !} \leqslant 0
$$


Выберем прямоугольньй контур $\Gamma$ с вершинами в точках $\pm i H, 2 \pm i H$. Очевидно выполнение неравенств

$$
\begin{array}{ll}
\operatorname{Re} f(s) \leqslant 0, & s \in \Gamma, \quad \sigma=0, \\
\operatorname{Re} f(s) \leqslant-1, & s \in \Gamma, \quad t= \pm H, \\
\operatorname{Re} f(s) \leqslant-\frac{2^{2 n}(4 n) !}{H^{4 n}}, & s \in \Gamma, \quad \sigma=2 .
\end{array}
$$

С другой стороны, имеем

$$
\operatorname{Re} f(1) \geqslant-\frac{73}{96} \frac{2^{2 n}(4 n) !}{H^{4 n}} .
$$

Рассмотрим теперь функцию

$$
F(s)=\zeta\left(\frac{1}{2}+i T+s\right) T^{f(s)}
$$

которая аналитична в области, ограниченной контуром $Г$. При $T \geqslant T_{2}>0$ на верхней и нижней сторонах Г имеем

$$
\left|\zeta\left(\frac{1}{2}+i T+s\right)\right| \leqslant \sqrt{T}
$$

откуда, пользуясь неравенством (9), находим

$$
|F(s)| \leqslant T^{-1 / 2}, \quad s \in \Gamma, \quad t= \pm H .
$$

На правой стороне контура Г имеет место оценка

$$
\left|\zeta\left(\frac{1}{2}+i T+s\right)\right| \leqslant \sum_{n=1}^{\infty} \frac{1}{n^{s / 2}} \leqslant 1+\int_{1}^{\infty} \frac{d u}{u^{s / 2}} \frac{5}{3},
$$

откуда в силу неравенства (10) получаем

$$
|F(s)| \leqslant \frac{5}{3} T^{-2^{2 n}(4 n) ! / H^{4 n}}, \quad s \in \Gamma, \quad \sigma=2 .
$$

В силу выбора $n$

$$
2^{2 n}(4 n) ! H^{-4 n} \leqslant\left(4 \sqrt{2} n H^{-1}\right)^{4 n} \leqslant e^{-4 n} \leqslant e^{-8},
$$

поэтому при $T \geqslant T_{3}>0$ будет

$$
T^{-1 / 2}<\frac{5}{3} T^{-2^{2 n}(4 n) ! H^{-4 n}}
$$

Таким образом, при $T \geqslant \max \left(T_{2}, T_{3}\right)>0$ справедлива оценка

$$
\max _{s \in \Gamma, \sigma>0}|F(s)| \leqslant \frac{5}{3} T^{-2^{2 n}(4 n) ! / H^{4 n}} .
$$


Пользуясь неравенством (11), находим

$$
|F(1)| \geqslant \frac{1}{3} T^{-(73 / 96)\left(2^{2 n}(4 n) ! / H^{4 n}\right)},
$$

ибо

$$
\left|\zeta\left(\frac{3}{2}+i T\right)\right|^{-1}=\left|\sum_{n=1}^{\infty} \frac{\mu(n)}{n^{3 / 2+i T}}\right| \leqslant \sum_{n=1}^{\infty} \frac{1}{n^{3 / 2}} \leqslant 1+\int_{1}^{\infty} \frac{d u}{u^{3 / 2}}=3 .
$$

В силу выбора $n$

$$
2^{2 n}(4 n) ! H^{-4 n} \geqslant\left(4 \sqrt{2} n H^{-1} e^{-1}\right)^{4 n} \geqslant\left(2 e^{2}\right)^{-4 n} \geqslant \exp \left(-\frac{3 H}{e \sqrt{2}}\right),
$$

ибо $[x] \geqslant x / 2$ при $x \geqslant 2$. Следовательно, при $T \geqslant T_{4}>0$ имеем

$$
\begin{aligned}
\frac{1}{3} T^{-(73 / 96)\left(2^{2 n}(4 n) ! / H^{4 n}\right)} & \geqslant \frac{5}{3} T^{-2^{2 n}(4 n) ! / H^{4 n}} \cdot \frac{1}{5} T^{(23 / 96) \exp (-(3 H) /(e \sqrt{2}))} \\
& >\frac{5}{3} T^{-2^{2 n}(4 n) ! / H^{4 n}}
\end{aligned}
$$

поскольку $H \leqslant \log \log T$ и $\exp \left((23 / 96)(\log T)^{1-3 /(e \sqrt{2})}\right)>5$ для достаточно больших $T$. Таким образом, при $T \geqslant \max \left(T_{2}, T_{3}, T_{4}\right)>0$ имеет место неравенство

$$
\max _{s \in \Gamma, \sigma>0}|F(s)|<|F(1)|
$$

откуда получаем

$$
|F(1)| \leqslant \max _{s \in \Gamma, \sigma=0}|F(s)| .
$$

Действительно, в противном случае имело бы место неравенство $|F(1)|>\max _{s \in \Gamma}|F(s)|$, которое противоречит принципу максимума модуля. Наконец, пользуясь неравенствами (8) и (12), имеем при $T \geqslant \max \left(T_{2}, T_{3}, T_{4}\right)>0$

$$
\max _{|t| \leqslant H}\left|\zeta\left(\frac{1}{2}+i T+i t\right)\right| \geqslant \max _{|t| \leqslant H}|F(i t)| \geqslant|F(1)| \geqslant \frac{1}{3} T^{-(73 / 96) \exp (-H /(2 e \sqrt{2}))} .
$$

Это завершает доказательство теоремы, поскольку для достаточно большого $T$ имеем

$$
\frac{1}{3} T^{-(73 / 96) \exp (-H /(2 e \sqrt{2}))}>T^{-\exp (-0.1 H)} .
$$

\section{СПИСОК ЦИТИРОВАННОЙ ЛИТЕРАТУРЫ}

[1] Карацуба А. А. О нижних оценках дзета-функции Римана // Докл. РАН. 2001. Т. 376. №1. C. $15-16$.

[2] Карацуба А. А. О нижних оценках максимума модуля $\zeta(s)$ в малых областях критической полосы // Матем. заметки. 2001. Т. 70. №5. С. 796-798.

[3] Garaev M.Z. Concerning the Karatsuba conjectures // Taiwanese J. Math. 2002. V. 6. № 4. P. 573-580.

[4] Balasubramanian R. On the frequency of Titchmarsh's phenomenon for $\zeta(s)$. IV // Hardy-Ramanujan J. 1986. V. 9. P. 1-10.

[5] Титчмарш Е.К. Теория дзета-функции Римана. М.: ИЛ, 1953.

[6] Прахар К. Распределение простых чисел. М.: Мир, 1967. 